\title{
Portal Vein Embolization: From Evidence to Expert Opinion?
}

\author{
Alban Denys • Thierry de Baere • Nicolas Demartines • \\ Pierre Bize
}

Received: 4 April 2012/ Accepted: 18 April 2012/Published online: 15 May 2012

(C) Springer Science+Business Media, LLC and the Cardiovascular and Interventional Radiological Society of Europe (CIRSE) 2012

Dr. Madoff's remarks address three different points. First, he addresses the difficult issue of extending the right portal vein embolization (PVE) to segment 4 branches in case of planned extended right hepatectomy. He states that we improperly cited his publication [6]. We agree that right PVE extended to segment 4 is not intended to hypertrophy the whole left liver (segments 2, 3, and 4). Dr. Madoff must have been confused by our use of "left lobe" of the liver, a term he seems to confuse with "left liver." Anatomic classification of liver segments as well as their name varies among different areas in the world. Nevertheless, in the most widely accepted textbook of liver surgery [1] as well as in most surgical centers, the left lobe is the part of the liver located medial to the falciform ligament-that is, segments 2 and 3 in the Couinaud nomenclature. Using these terms, our sentence is correct and properly reflects Dr. Madoff's own statement. Dr. Madoff argues that his publication does not demonstrate that segment 4 embolization improves the hypertrophy rate of the left lobe. However, in the same reference [6], Dr. Madoff writes in the discussion that "the experience reported herein highlights...the advantage of segment 4 embolization and the advantages of embolization with small spherical particles used in combination with coils." Despite his own

A. Denys $(\bowtie) \cdot$ P. Bize

Department of Radiology and Interventional Radiology, Lausanne University Hospital, 1011 Lausanne, Switzerland e-mail: Alban.Denys@chuv.ch

T. de Baere

Department of Interventional Radiology, Institut Gustave Roussy, 39 Rue Camille Desmoulins, 94800 Villejuif, France

N. Demartines

Department of Visceral Surgery, Lausanne University Hospital, 1011 Lausanne, Switzerland statement, we recognize, as mentioned in his letter, that this retrospective study more clearly reflects the potential benefit of small particles over nonspherical ones, rather than the benefit of segment 4 branches embolization. In another sentence, he recognizes that segment 4 branch embolization needs "extreme care to avoid reflux in segments 2 and 3 branches,... and may require substantially more time and contrast than right PVE." In another article, surprisingly, segment 4 increased in volume after right +4 PVE ( $+26 \%$ similarly to right PVE $+20 \%$ ); this finding might reflect the difficulty in recognizing all segment 4 branches and/or completely occluding all of them [5]. Other centers reviewing the largest series published to date have not found a difference in hypertrophy of the future remnant liver between these two techniques [3]. The final answer to this point should be provided by randomized trial, but in the meantime, we recommend that segment 4 branch embolization should be performed only by experienced interventional radiologists and when anatomy is appropriate (large segment 4 branches) using easily controllable embolic material.

Second, Dr. Madoff comments on the choice of the embolic agent for PVE. Nearly all commercially available embolic agents have been used for PVE and produced, more or less, left liver hypertrophy after right PVE. From the patient's perspective, the choice of the "best" embolic agent should be according to whether it performs better at inducing higher hypertrophy of the future remnant liver, whether it has a lower rate of complications but works similarly, or both. The authors state that there is only one "small animal study" comparing NBCA ( $n$-butyl-cyanoacrylate) to spherical particles, thus supporting the use of NBCA versus spherical particles in terms of performance [2]. Recently, another experimental publication demonstrated the same results [8] when comparing NBCA to 
small particles in an animal model using more recent evaluation technologies. It is interesting, as we point out in our review, that regeneration of the future remnant liver seems correlated to the periportal inflammation created in the embolized liver by the embolic material [2]. This has been demonstrated both in animals and in humans, and this strongly favors the use of NBCA as compared to particles, coils, and plugs, because NBCA induces little or no periportal inflammation. Concerning the complication rate, the only large series that specifically addressed this point was performed on patients embolized with NBCA [4]. To our knowledge, no series including the same number of patients and using particles specifically addressed this point. Nevertheless, severe complications like left portal vein thrombosis that can preclude further liver resection are observed despite an ipsilateral approach and the use of particles [7]. Dr. Madoff's citations of abstracts adds little to this debate because these series have not been published, making their detailed analysis impossible.

Finally, Dr. Madoff comments on the benefit of an ipsilateral versus contralateral approach to the portal tree. Each approach carries both risks and advantages. It is also related to the choice of the embolic material and to the anatomic territory that needs to be treated. In a recent review, van Gulik et al. [9] quoted a survey conducted in the Netherlands during 2006-2007. During this period, only 98 portal occlusions were performed in this country, only half of them by means of percutaneous embolization-the result of a lack of experienced interventional radiologists. This honest statement clearly establishes that PVE is a technique performed probably less than once a month even in large centers, and each interventional radiologist must thus feel confident with the method that he or she uses. In our review, we wanted to honestly present the benefits and drawbacks of each access route. Practitioners can then make an informed choice.

Dr. Madoff's contributions to the field of PVE are impressive, as is his work with Nicolas Vauthey, a liver surgeon at the MD Anderson Cancer Center, Houston,
Texas. They have made a lot to promote this technique that was invented in Japan and modified in Europe. We thus expect that most of the questions his remarks raise will find more robust response than only expert opinion.

Conflict of interest The authors declare that they have no conflict of interest.

\section{References}

1. Blumgart L, Hann L (2007) Surgical, and radiological anatomy of the liver, biliary tract, and pancreas. In: Blumgart L (ed) Surgery of the liver, biliary tract, and pancreas. Elsiever, Philadelphia, pp 3-30

2. de Baere T, Denys A, Paradis V (2009) Comparison of four embolic materials for portal vein embolization: experimental study in pigs. Eur Radiol 19:1435-1442

3. de Baere T, Teriitehau C, Deschamps F et al (2010) Predictive factors for hypertrophy of the future remnant liver after selective portal vein embolization. Ann Surg Oncol 17:2081-2089

4. Di Stefano DR, de Baere T, Denys A et al (2005) Preoperative percutaneous portal vein embolization: evaluation of adverse events in 188 patients. Radiology 234:625-630

5. Kishi Y, Madoff DC, Abdalla EK et al (2008) Is embolization of segment 4 portal veins before extended right hepatectomy justified? Surgery 144:744-751

6. Madoff DC, Abdalla EK, Gupta S et al (2005) Transhepatic ipsilateral right portal vein embolization extended to segment IV: improving hypertrophy and resection outcomes with spherical particles and coils. J Vasc Interv Radiol 16(2 pt 1):215-225

7. Madoff DC, Hicks ME, Abdalla EK, Morris JS, Vauthey JN (2003) Portal vein embolization with polyvinyl alcohol particles and coils in preparation for major liver resection for hepatobiliary malignancy: safety and effectiveness-study in 26 patients. Radiology 227(1):251-260

8. Tsoumakidou G, Theocharis S, Ptohis N et al (2011) Liver hypertrophy after percutaneous portal vein embolization: comparison of N-butyl-2-cyanocrylate versus sodium acrylate-vinyl alcohol copolymer particles in a swine model. Cardiovasc Intervent Radiol 34:1042-1049

9. van Gulik TM, van den Esschert JW, de Graaf W et al (2008) Controversies in the use of portal vein embolization. Dig Surg 25:436-444 